\title{
Socio-Political Emotional Climate of Turkey and Its Effects: A Qualitative Examine
}

\section{BACKGROUND}

It is possible to assume that the sociopolitical structure, which is one of the systems surrounding the individual, may have individual effects in the context of mental health and functionality. Emotional climate is one of the characteristics that may originate from socio-political conditions. It refers to the predominant collective emotions generated through the social interaction between members of society and socialpolitical factors in a particular milieu (de Rivera, 1992, 2007). In this study, it is aimed to determine the collective emotions, the socio-political factors associated with these emotions, and how these emotions affect individuals subjectively.

\section{METHOD}

The sample of the study consists of eight people who are satisfied/dissatisfied about the current political system and course, as well as young and middle age and low and high education groups. For the purpose of the study, semi-structured interviews were conducted that asked the emotions about living in Turkey, emotions toward to other people in the community, psychosocial factors about these emotions and their individual effects, The contents were analyzed via MAXQDA 18 by creating upper and sub codes. Coding within a general structure method was used.

\section{FINDINGS}

\section{Collective Emotions}

As a result of analyses, it was found that most common collective emotions were anger, worry, sadness, fear, oppression, resentment, polarization.

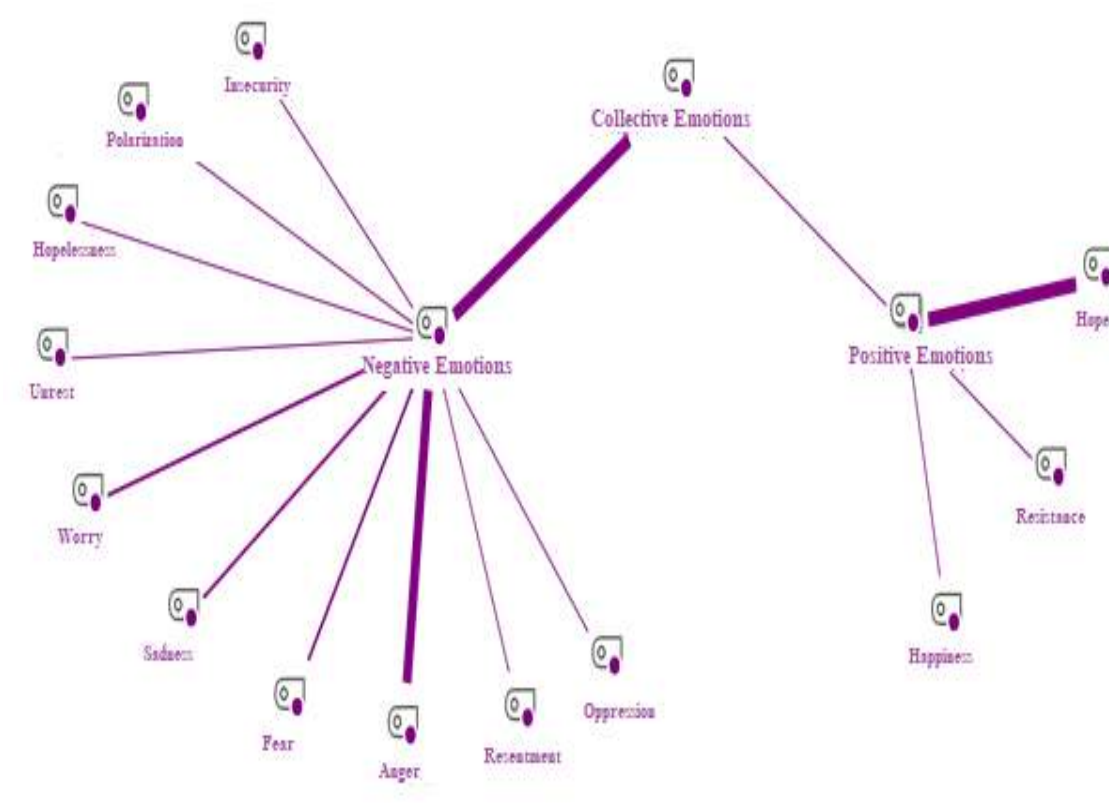

\section{Feelings Toward to Other People}

It was seen that the feelings towards other people in the community were predominantly negative due to the perception of ostracizing, oppression and inequality.

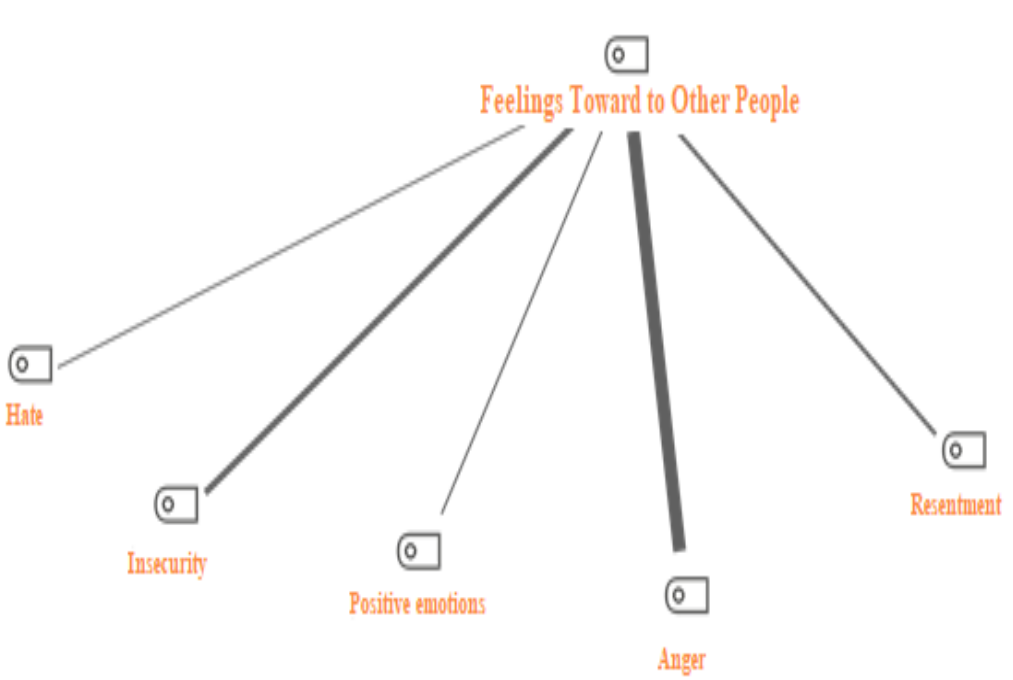

\section{The Factors that Cause Collective Emotions}

The factors that give rise to collective emotions were grouped in injustice unpredictability, problems in social policies, violence, threat against the existence and onnression.

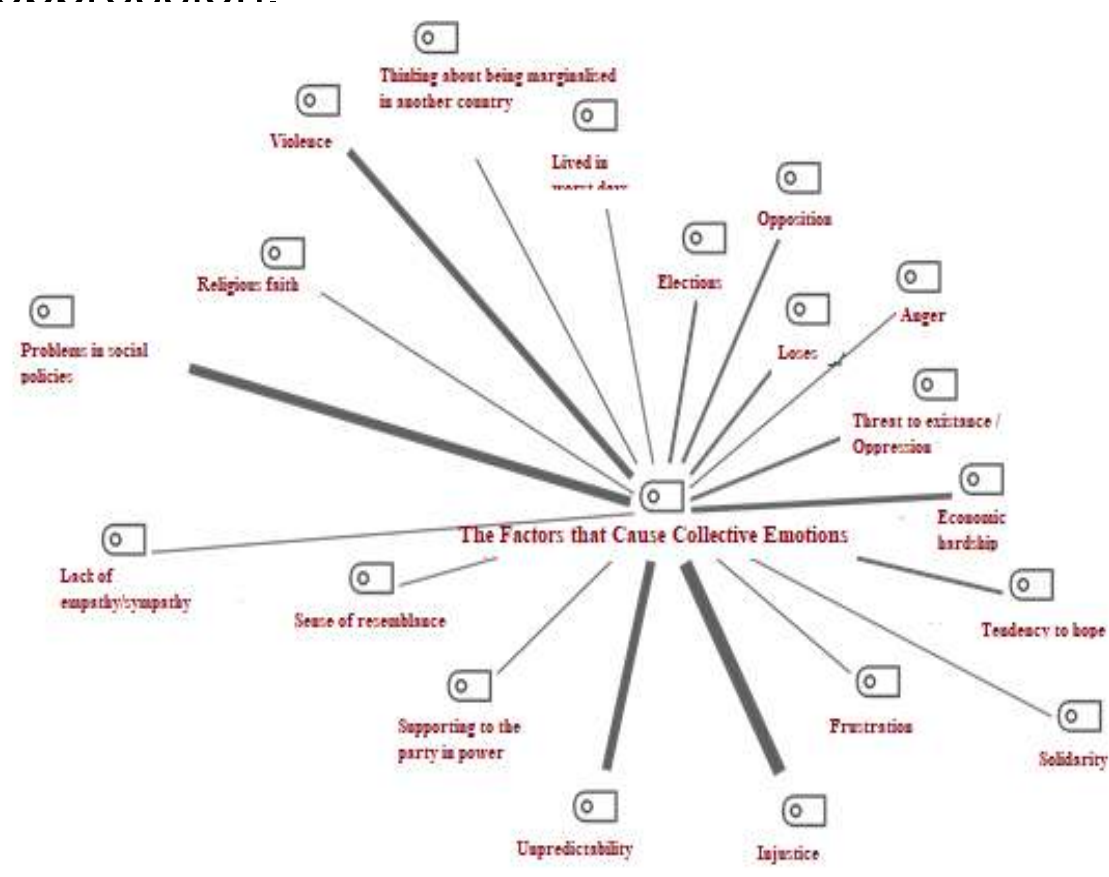

\section{Individual Effects of Collective Emotions}

The individual effects of these collective emotions and their sources were some feelings such as anger, numbness, resistance and also, desire to isolation, positive contribution to identity formation, preoccupation with political issues.

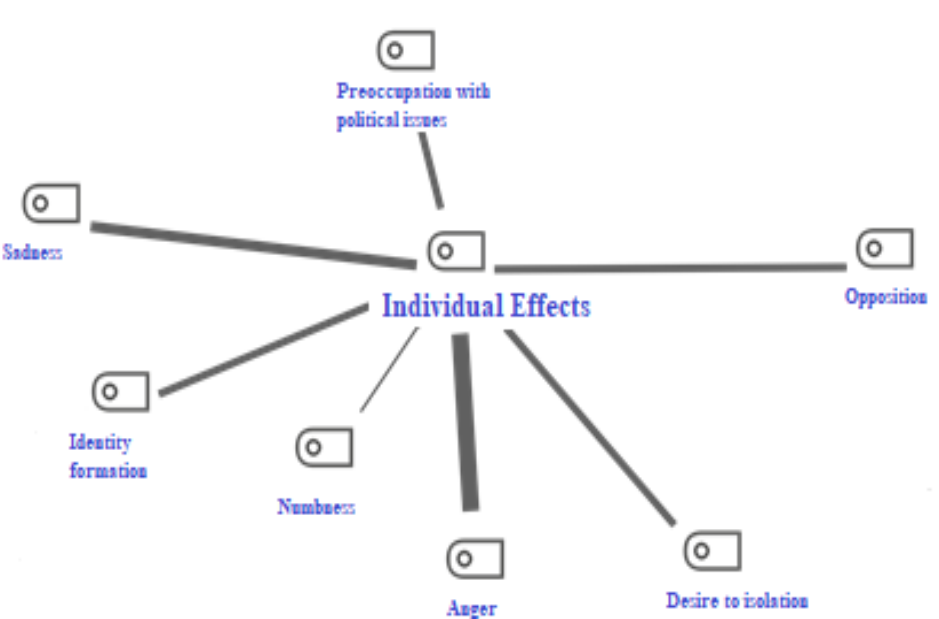

\section{DISCUSSION}

Mental health is an integrated situation which contains emotional, psychological and social well-being (Keyes, 1998, 2005). The findings of this study indicate that socio-political factors affect well-being and functionality in various ways. Studies in this area contribute to the establishment of mental health in the social framework and provide evidence for social policies to protect mental health and to prevent psychological disorders.

\section{REFERENCES}

de Rivera, J. (1992). Emotional climate: Social structure and emotional dynamics. KT Strongman (Eds.), International review of studies on emotions.

de Rivera, J., ve Páez, D. (2007). Emotional climate, human security, and culture of peace. Journal of Social Issues, 63 , 233-253.

(1998). Social well-being. Social Psychology Quarterly, 121-140.

Keyes, C. L. (2005). Mental illness and/or mental health? Investigating axioms of the complete state model of health. Journal of Consulting and Clinical Psychology, 73, 539-548. 